\title{
Molecular Mechanism of in Vitro Oligomerization of Dps from Mycobacterium smegmatis: Mutations of the Residues Identified by "Interface Cluster" Analysis ${ }^{\dagger}$
}

\author{
Rakhi Pait Chowdhury, ${ }^{\ddagger}$ M. S. Vijayabaskar, ${ }^{\ddagger}$ Saraswathi Vishveshwara, and Dipankar Chatterji* \\ Molecular Biophysics Unit, Indian Institute of Science, Bangalore-560012, India
}

Received June 20, 2008; Revised Manuscript Received August 22, 2008

\begin{abstract}
The irreversible dodecamerization of native Dps trimers from Mycobacterium smegmatis, in vitro, is known to be directly associated with the bimodal function of this protein. Hence it is important to explore this pathway at the molecular level. Two types of trimers, Trimer A (tA) and Trimer B (tB), can be derived from the dodecamer due to the inherent 3-fold symmetry of the spherical crystal structure. These derived trimers were expressed as protein structure graphs (PSGs) using the computed interaction strength among the residues. Interface clusters which were identified from PSGs allowed us to convincingly predict E146 and F47 for further mutation studies. Various single and double mutants were constructed and characterized. We were finally able to generate a single mutant F47E impaired in dodecamerization and a double mutant E146AF47E as native monomer in solution. These two observed results suggest that the two trimers are important for dodecamerization and that the residues selected are important for the structural stability of the protein in vitro.
\end{abstract}

Symmetry plays an important role toward folding of a multi-subunit protein in vitro $(1-3)$. It reflects the multiplicity at the interface contacts and hence the number and position of required mutation(s) to disrupt the interfaces. In most of the cases it has been seen that the thermal folding kinetics follows an irreversible path and most probably the kinetic barrier for the reverse path is too high to overcome, which makes the multimeric assembly highly stable in solution (4). Hence, it becomes relevant to explore the structural determinants that drive the assembly of such identical monomeric subunits to form a highly symmetric stable oligomer. This will open new molecular insights on the relative significance of each interaction present at the oligomeric interface(s) stabilizing the structure.

Different techniques have been utilized in the past $(5-8)$ to elucidate the mechanism of protein assembly and concomitant function. Here, we are interested in correlating the quarternary structure of a protein and its biological function. The DNA binding protein from starved cells (Dps) of Mycobacterium smegmatis is well-characterized in vitro from both structural (9-11) and functional (12) aspects. This novel starvation inducible protein is unique from the rest of the members of the family with respect to the temperature induced irreversible oligomeric transition from a trimer to a dodecamer in solution, where the lower oligomer is capable of inhibiting the Fenton's reaction and thus protecting the

\footnotetext{
${ }^{\dagger}$ This work was supported by Grant Part IIB Department of Biotechnology (DBT) infrastructure program support for basic biological research. R.P.C and M.S.V are funded by a senior research fellowship from the Council of Scientific and Industrial Research and Indian Institute of Science, Government of India, respectively.

* Corresponding author. Address for correspondence: Molecular Biophysics Unit, Indian Institute of Science, Bangalore-560012, India. Phone: +91-80-2293-2836. Fax: +91-80-2360-0535. E-mail: dipankar@ mbu.iisc.ernet.in.

${ }^{\ddagger}$ Both the authors have equal contribution to this work.
}

bacterial DNA from toxic hydroxyl radicals. Along with this property, a physical shielding of the DNA is also provided through a nonspecific binding of only the dodecameric form, which is named as "bimodal function" of Ms-Dps protein (12). Thus, the oligomeric transition is directly associated with this bimodal functionality of the protein itself, which is essential for the bacteria to survive under the stressful conditions inside the host at the stationary phase. The crystal structure of the protein reveals that it is a symmetric dodecamer, and two different trimers can be deduced, namely "Trimer A (tA) and "Trimer B" (tB) (4), due to the inherent $C_{3}$ symmetry. Our aim is to elucidate the molecular mechanism of this oligomerization and therefore to probe the important amino acid residues at the trimeric interface regions.

In the present work, we have used graph theoretical method $(13-15)$ to predict the important amino acid residues, situated at the interface regions which stabilize the oligomeric structure with side chain interactions (16-19). All the factors such as the hydrophobic core (20), electrostatic (21) and aromatic stacking (22) contributing to the interface stability are effectively captured as interface clusters by this graph based method. This algorithm considers global nonbonded interactions among the amino acids in the form of a mathematical graph represented as an adjacency matrix (see Materials and Methods) and is very well established in identifying clusters of residues significantly contributing to the stabilization of the protein oligomeric structure $(15,19)$. The amino acid(s), identified as crucial for oligomerization, are then subjected to site directed mutagenesis $(13,14,23)$ to an amino acid distinct from both stereochemical and physical aspects. These mutants are then characterized structurally along with their ability to oligomerize and bind DNA. In the present study, two residues, E146 from tA and F47 from tB interface clusters, are identified as crucial for 
stabilizing the Dps trimers. We show here that F47 residue is crucial for dodecamerization.

\section{MATERIALS AND METHODS}

Identification of Interface Clusters. A protein structure graph $\left(\mathrm{PSG}^{1}\right)(15)$ is defined as nodes connected by edges, in which the nodes are the amino acids in the protein. The edges are drawn on the basis of the interaction strength between the nodes. Interaction strength $\left(I_{i j}\right)$ between any two nodes " $i$ " and " $j$ " is defined as

$$
I_{i j}=\frac{n_{i j}}{\sqrt{N_{i} \times N_{j}}} \times 100
$$

where $n_{i j}$ is the number of unique atom pairs between " $i$ " and " $j$ " within a distance of $4.5 \AA$.

$N_{x}$ is the normalization value for the residue type " $x$ ". Here, we should note that we have considered normalized interactions as a measure of the interaction strength among amino acids.

An adjacency matrix $\mathbf{A}$ is defined with every element of the matrix satisfying the below rule.

$$
A_{i j}=\left\{\begin{array}{l}
1, I_{i j}>0 \text { and } I_{i j} \geq I_{\min } \\
0, I_{i j}=0 \text { or } I_{i j}<I_{\min }
\end{array}\right.
$$

where $I_{\min }$ is the minimal interaction strength. If $A_{i j}=1$, then the nodes " $i$ " and " $j$ " are connected in the graph. Hence the topology of connectivity in a PSG is given by the adjacency matrix. Using this PSG or the adjacency matrix, properties like clusters and hubs can be identified. A cluster is defined as a group of interacting amino acid nodes such that there are no edges from the cluster nodes, incident on other noncluster nodes. Here the DFS (depth first search) algorithm was used to identify clusters (24). A cluster is termed as an interface cluster if the participating nodes are from different monomers of the multimeric complex. Hubs are highly connected nodes in a network and are considered to be vital in maintaining the stability of networks $(25-29)$. A hub is declared as an interface hub if the edges incident on it comes from at least one node of the other monomers.

Site Directed Mutagenesis of the Dps Gene. The M. smegmatis $d p s$ gene was cloned in an expression vector pET21b in Escherichia. coli, and this was used as the template for site directed mutation(s) of one or more amino acid at the interface. Mutagenesis was carried out using the Quickchange protocol (Stratagene) and single primer method (23). The primers for generating all the single and double mutants at different oligomeric interfaces are listed in Table 1. All the mutants were confirmed by DNA sequencing and MALDI-TOF mass spectrometry of the expressed proteins.

Expression and Purification of the Dps Protein and Its Single and Double Mutants. The C-terminal His-tagged single and double amino acid mutants of Dps (see Table 2 for all the developed mutants and the nomenclature we use to denote these mutants) were purified following a similar protocol as for the wild type protein (12). E. coli strain BL21

\footnotetext{
${ }^{1}$ Abbreviations: MALDI-TOF-MS, matrix assisted laser dissociation ionization time of flight mass spectrometry; $\mathrm{CD}$, circular dichroism; DLS, dynamic light scattering; MW, molecular weight; aa, amino acid; PSG, protein structure graph; BSA, bovine serum albumin; PAGE, polyacrylamide gel electrophoresis.
}

Table 1: Primers Used for Generation of Single and Double Mutants at Different Interface Clusters

\begin{tabular}{lll}
\hline mutations & $\begin{array}{c}\text { primer } \\
\text { name }\end{array}$ & \multicolumn{1}{c}{$\begin{array}{c}\text { primer sequence } \\
\left(5^{\prime}-3^{\prime}\right)\end{array}$} \\
\hline E146A & dE146A & CCGGCGAGCTGGCGAAATTCCAGTGGTTCG \\
& dE146Ar & CGAACCACTGGAATTCGCCAGCTCGCCGG \\
F47E & dF47E & GTCGTGGGCCCGAACGAGATCGGCGTGCACG \\
& dF47Er & CGTGCACGCCGATCTCGTTCGGGCCCACGAC \\
E47Y & E47Yf & TGGGCCCGAACTACATCGGCGTGCACG \\
& E47Yr & GCTGCACGCCGATGTAGTTCGGGCCCA \\
\hline
\end{tabular}

Table 2: Construction of Dps Mutants through Single or Double Amino Acid Mutations

\begin{tabular}{llc}
\hline \multicolumn{1}{c}{ protein } & \multicolumn{1}{c}{ mutations } & nomenclature \\
\hline trimer A (tA) interface single mutant & E146A & tAm \\
trimer B (tB) interface single mutant & F47E & tBm \\
trimer B (tB) interface reverse mutant & E47Y & tBmR \\
double mutant at trimeric clusters & E146AF47E & DM \\
\hline
\end{tabular}

DE3 (pLys) was transformed with the vector (pET21b) carrying the mutant clones. These cells were grown at 37 ${ }^{\circ} \mathrm{C}$ in Luria Bertini (LB) medium to an $A_{600}$ of 0.6 and then induced with $1 \mathrm{mM}$ isopropyl-1-thio- $\beta$-galactopyranoside. Single step purification was performed using the Qiagen NiNTA affinity matrix according to the manufacturer's instructions. The protocol was little modified with respect to an increased $\mathrm{NaCl}$ concentration in the buffers $(1 \mathrm{M})$ while purifying double mutant (DM). The purification profile of all the proteins was checked on a 12\% SDS-polyacrylamide gel, and then the protein was dialyzed against $50 \mathrm{mM}$ Tris$\mathrm{HCl}\left(\mathrm{pH} \mathrm{7.9,} 4{ }^{\circ} \mathrm{C}\right), 150 \mathrm{mM} \mathrm{NaCl}$ for overnight and oligomeric status of all the native proteins were checked on a $10 \%$ native-PAGE. For the formation of the dodecamer, proteins at a concentration of $>1 \mathrm{mg} / \mathrm{mL}$ were incubated at $37{ }^{\circ} \mathrm{C}$ for $12 \mathrm{~h}$ in $50 \mathrm{mM}$ Tris- $\mathrm{HCl}\left(\mathrm{pH} 7.9,4{ }^{\circ} \mathrm{C}\right), 150 \mathrm{mM}$ $\mathrm{NaCl}$ (12). All the proteins were subjected to MALDI-TOF mass spectrometry in order to verify the expected mass, hence confirming the correct number of mutations.

Structural and Functional Characterization of the Dps Mutants. The purified Dps protein and all its mutants were subjected to Superdex 75 HR 10/30 gel filtration column (Pharmacia) at a slow flow rate of $0.25 \mathrm{~mL} / \mathrm{min}$, in a buffer containing $50 \mathrm{mM}$ Tris- $\mathrm{HCl}\left(\mathrm{pH} 7.9,4{ }^{\circ} \mathrm{C}\right), 150 \mathrm{mM} \mathrm{NaCl}$. High molecular weight native protein marker (Fermentas) was used for molecular weight calibration. Molecular weights and oligomeric status were also confirmed from MALDITOF-MS and DLS experiments. Finally mutations were all confirmed by DNA sequencing. The secondary structures of the proteins were also verified by performing near and far UV CD experiments. The native and mutants were all characterized with respect to ferroxidation activity, iron binding and DNA binding $(9,12)$.

\section{RESULTS}

The crystal structure of Ms-Dps dodecamer was resolved at $4.0 \AA$ resolution in its hexagonal form $(10,11)$. The protein monomer (183 aa) was found to be a four helix bundle (chains A, B, C, D) consisting of $\sim 65 \% \alpha$ helical structure connected through intermediate loops. However, upon purification of the His-tagged recombinant protein it resolves as a trimer in both PAGE as well as gel filtration chromatography experiments. Both the N-terminal (1-10 aa) and a 
(a)

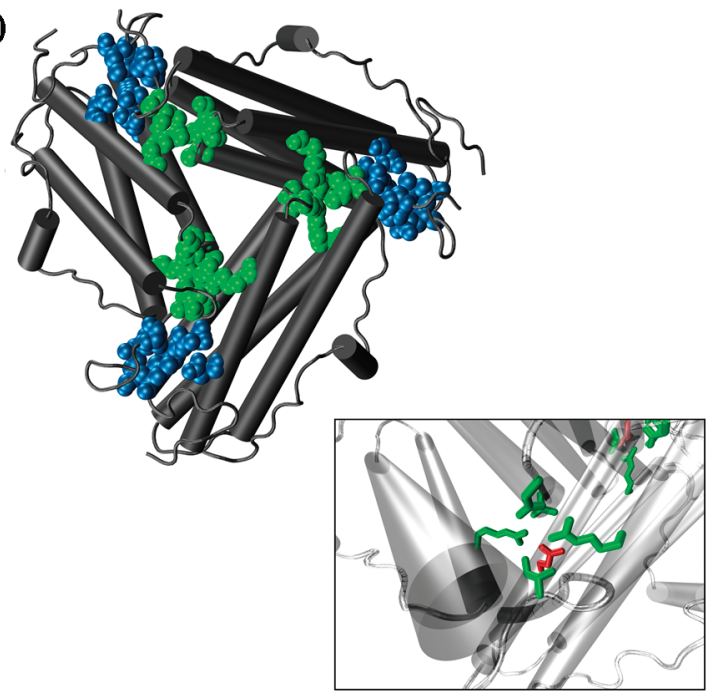

(c)

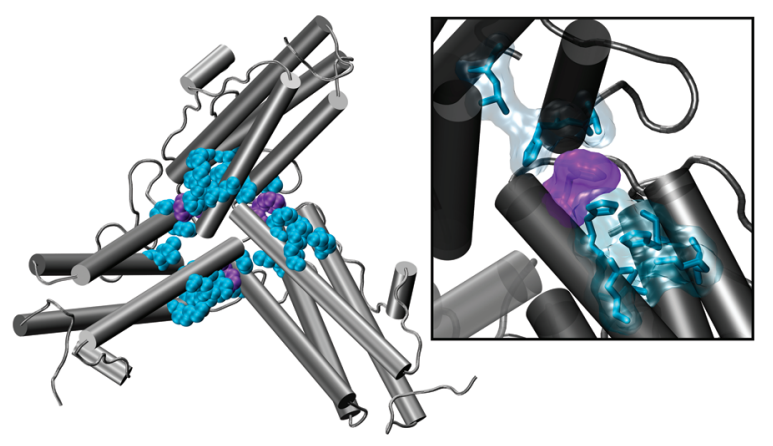

(b)

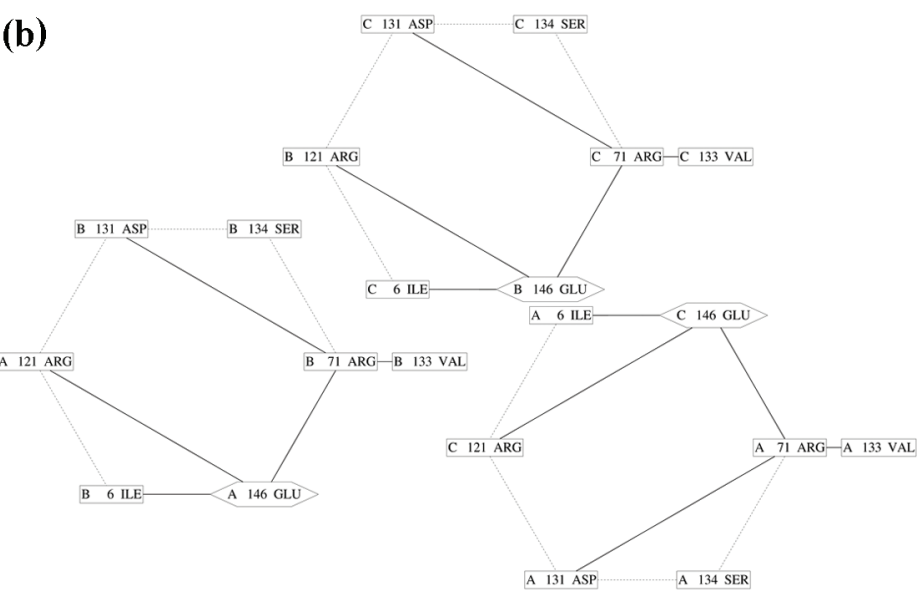

(d)

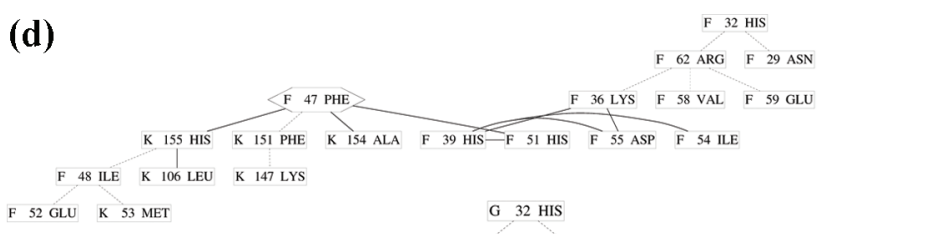

G 29 ASN G 62 ARG

F 155 HIS F 154 ALA G 51 HIS F 151 PHE G 39 HIS - G 55 ASP G 54 ILE

G 48 ILE F 106 LEU

F 147 LYS

F 53 MET G $52 \mathrm{GLU}$

K 32 HIS

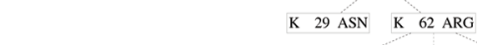

K 47 PHE $\quad$ K 58 VAL K 59 GLU K 36 LYS

G 155 HIS G 154 ALA K 51 HIS G 151 PHE K 39 HIS-K 55 ASP K 54 ILE

K 48 ILE G 106 LEU

G 147 LYS

G 53 MET K $52 \mathrm{GLU}$

FIGURE 1: Prediction of interface clusters in the Dps trimers from graph theory. (a) Trimer A (tA) interface obtained from the dodecamer crystal structure. Protein-protein interface cluster residues are represented using VDW representation with colors indicating the chain from which the residues form clusters. Inset: The salt bridge formed by the four residues is being highlighted. The predicted mutation site E146 is highlighted in red. (b) The subgraph of the salt bridge interface cluster (at $I_{\min }=4 \%$ ) in tA is shown, demonstrating the connectivity among the amino acid residues. The highly interacting pairs $\left(I_{i j} \geq 7 \%\right)$ of amino acids are connected through solid lines, and the other interactions that vanish on increasing the $I_{\min }$ (from $4 \%$ to $7 \%$ ) are shown in dotted lines. (c) Trimer B (tB) is shown with cluster residues highlighted as VDW. Inset: The hydrophobic interactions of the predicted mutation site residue F47 (highlighted as violet) is shown. (d) The interface cluster (at $I_{\min }=4 \%$ ) in $\mathrm{tB}$ is shown as a connectivity graph. The solid and the dotted lines imply the same as mentioned in (b).

long C-terminal tail (157-183 aa) are found to be exposed toward the surface, where the latter appeared to be responsible for the DNA binding ability of this protein $(12,30)$. Thus, the novelty of Ms-Dps, so far, as characterized by the DNA binding activity of the dodecameric form is confined to the surface of this spherically symmetric molecule. The protein converts from a trimer to a dodecamer upon temperature induction from 4 to $37{ }^{\circ} \mathrm{C}$ under optimum buffer conditions and protein concentration. Here we are interested at the "interface" region, and construct some mutant(s) of Dps protein, which are locked in monomeric or trimeric form. This exercise will reveal the factors contributing to the pathway of oligomerization and the associated stability. In this study, we have used graph theoretical methods, in which the protein is expressed as protein structure graphs (PSGs), to identify probable mutation points. PSGs offer an advantage over conventional methods in various ways. They are computed taking into account the residue-wise contacts, thus generating relationship between residues as a function of interaction strength, and at the same time provide valuable information on the connectivity pattern among residues. This quantification of the interactions and the connectivity pattern help to narrow down the structurally important residues with logical clarity.

Cluster Locations and Residue Identification for Mutation. PSGs of globular proteins were found to follow a sigmoidal profile as a function of $I_{\min }$. The $I_{\min }$ in the transition phase is termed as $I_{\text {critical }}(15)$. An optimal region of $I_{\min }$ for analyzing the PSGs is around this $I_{\text {critical }}$. Hence, we went ahead to analyze the cluster around this $I_{\text {critical }}$ region, which corresponded to an interaction strength of $I_{\min }=4 \%$. The two trimers of Dps were expressed as PSGs, as described in Materials and Methods, and the interface clusters were then identified. We found that the two trimers lacked a true trimeric interface cluster but were composed of symmetrically positioned dimeric interface clusters (Figure 1). Hence a trimer consists of three identical interface clusters, with each cluster comprising any two of the three monomer chains.

(i) Clusters in tA. At lower $I_{\min }$ values $(0 \%$ to $4 \%$ ) we find a single large cluster forming at the dimeric interfaces 
of tA. However, as we increase the $I_{\min }$ the large cluster breaks up into two distinct clusters (Figure 1a, shown in "blue" and "green"). This implies that the interaction mechanism in tA is governed by two distinct clusters (group of residues) capable of stabilizing the trimer autonomously. One of the clusters has very strong salt bridge interactions (shown in "green" in Figure 1a), compared to the other cluster which is a weak interface cluster (This cluster vanishes at $I_{\min }>4 \%$ ). The salt bridge cluster is large at lower $I_{\min }$ values, and as we increase the $I_{\min }(>5 \%)$, the size of the cluster diminishes by losing weakly connected amino acid nodes (shown in "dotted" lines in Figure 1b), and only the stronger interactions are retained (as shown by "solid" lines in Figure 1b). Out of these residues, residue E146 is an "interface hub", connected through strong interaction network with the amino acids of the other chain (Figure 1b). This is also one of the residues with almost complete buried surface area upon trimerization. Hence we have speculated that an E146A mutation may result in the disruption of salt bridges and, hence, the formation of tA interface. It should be noted here that the connectivity pattern generated here suggests two probable mutation points, indicated as Arg 121 and Glu 146. However, Glu 146 stands apart from the point of interacting strength. Any conventional analysis may overlook this point.

(ii) Clusters in $t B$. Unlike tA, tB has a single unique cluster dominated by aromatic stacking and hydrophobic interactions at the interface (Figure 1c, shown in "blue"). At lower $I_{\min }$ values $(\leq 4 \%)$ the cluster is large and single, and as we increase the $I_{\min }$, the cluster remains single but the size diminishes by losing weakly connected (shown by dotted lines in Figure 1d) amino acid nodes. From Figure 1d, it is clear that only F47, at the interface cluster, interacts directly with the residues of the other chain. Additional data show that F47 is also one of the completely buried residues and also the only "interface hub". Hence we predicted it as a potential mutation point (Figure 1c, shown in "violet"). By mutating F47 to E47 we were able to completely disrupt the tB trimer (experimental confirmation will be discussed in a later section) by destabilizing the hydrophobic/aromatic interactions at the interface. The mutations that were created at the interface (as presented above), predicted from PSGs, are shown in Table 2.

Single Amino Acid Mutants tAm and tBm of Dps: tBm Cannot Form Dodecamer in Vitro. It has been recently shown that disruption of a single salt bridge interaction located at a subunit interface in Listeria innocua Dps (LiDps) converted it into the Dps from its facultative pathogen Listeria monocytogenes (LmDps). Through mutational analysis at the interface they have conferred the significance of optimized surface electrostatics and hydrogen bonding toward stabilization of a multimeric protein assembly (4). LmDps is considered to be a natural mutant of LiDps, as it differs in only two amino acid residues Gln114 and Asn126 forming hydrogen bonds compared to Lys114 and Asp126 involved in salt bridge interaction in LiDps.

The salt bridge cluster in tA form of Dps is composed of strong electrostatic and ionic interactions (Figure 1b), and hence it was reasonable to expect that the mutation of the 146th glutamate residue to alanine probably would contribute toward the multimeric protein assembly as well as the thermodynamic and kinetic stability of the oligomeric form

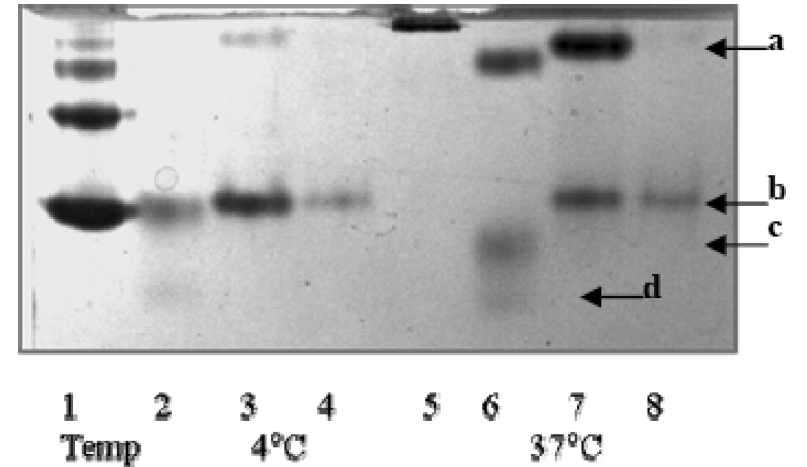

FIGURE 2: $10 \%$ native-PAGE profile of oligomerization of native Dps and the single mutants. BSA (lane 1); native Dps (lane2); tAm (lane 3); tBm (lane 4); horse spleen ferritin (lane 5); native Dps (lane 6); tAm (lane 7); tBm (lane 8). Here "a" represents the higher oligomer (dodecamer), "b" represents the lower stable oligomer (trimer) and "c" and "d" represent the possible dimeric and monomeric forms respectively present in solution during dodecamerization of the native form.

in solution. The choice of E146 as a potential mutation point over other residues in the network is discussed in the previous section. However, the tAm mutant, upon purification, did not alter the oligomeric assembly as it resulted in a trimer and was also further capable of dodecamerization under similar condition to that of the native protein (Figure 2, lanes $2,3,6,7)$. This might possibly be because of the presence of two autonomous clusters. Hence, the tA structure remained intact either due to stabilization through the other cluster or perhaps due to partial disruption of salt bridges or both. Native Dps and tAm showed certain interesting difference on $10 \%$ native-PAGE. In the former case there were lower molecular weight bands which were absent in the latter (compare lanes 6 and 7 in Figure 2, bands "c" and "d"). This will be elaborated further in the discussion part.

On the other hand, the tB interface clusters, as shown in Figure 1c and d, provided unique hydrophobic side chain interactions among the neighboring residues where the 47th phenyl alanine is significantly placed as a "hub" in the amino acid network. By mutating this to glutamate, so as to completely disrupt the hydrophobic property of the amino acid, we generated a mutant $\mathrm{tBm}$, which forms a stable trimer upon purification at $4{ }^{\circ} \mathrm{C}$ (Figure 2, lane 4). However, this mutant was unable to form dodecamer (Figure 2, lane 8), under similar condition like that of native Dps and tAm. All the mutants were subjected to DLS as well as CD experiments (see Supporting Information Table 1B and Figures $\mathrm{S} 1$ and $\mathrm{S} 2$ respectively) to characterize them with respect to their oligomeric status and secondary structure stability, which gave similar results as that obtained in the case of native protein, retaining the fold. This was then followed by functional characterization of the mutants with respect to ferroxidation activity, iron binding and DNA binding ability (see Supporting Information Table 2 and Figures S3-S5).

Reverse Mutagenesis of the 47th Amino Acid in tBm from "E" (Glutamate) to " $Y$ " (Tyrosine) Restored Dodecamerization. To further confirm our prediction that F47 residue was important for dodecamerization, we performed reverse mutagenesis with a new set of primers (Table 2), at this 47th position from $\mathrm{E}$ to $\mathrm{Y}$ to reintroduce the lost hydrophobic/ aromatic interactions in the tBm trimer. This finally generated 


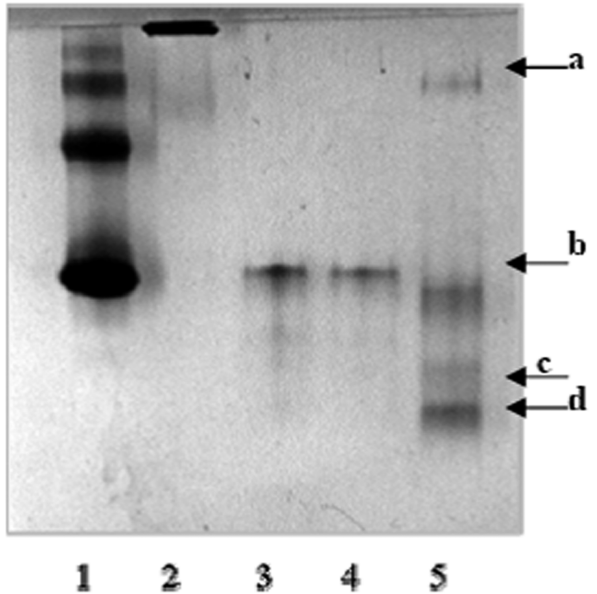

FIGURE 3: The dodecamerization property of $\mathrm{tBm}$ mutant is restored upon mutation of the 47th amino acid from glutamate (E) to tyrosine (Y). BSA (lane 1); horse spleen ferritin (lane 2); tBmR (trimer) (lanes 3, 4); tBmR (dodecamer) (lane 5). Here "a" represents the higher oligomer (dodecamer), " $b$ " represents the lower stable oligomer (trimer) and "c" and "d" represent the possible dimeric and monomeric forms respectively present in solution during dodecamerization of the native form.

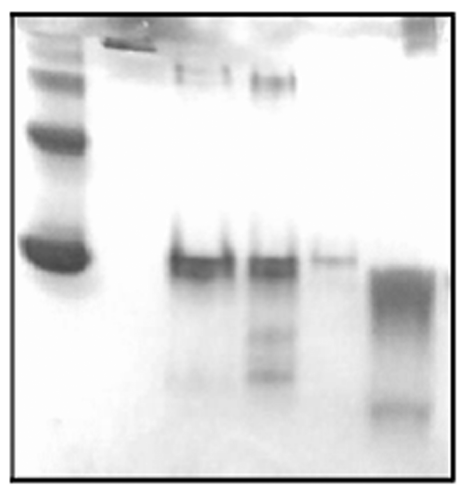

\section{$\begin{array}{llllll}1 & 2 & 3 & 4 & 5 & 6\end{array}$}

FIGURE 4: Purification profile (10\% native-PAGE) of native Dps and the trimeric interface mutants $\mathrm{tAm}, \mathrm{tBm}$ and $\mathrm{DM}$ under $0.5 \mathrm{M}$ salt conditions at $4{ }^{\circ} \mathrm{C}$ : BSA (lane 1); horse spleen ferritin (lane 2); native (lane 3); tAm (lane 4); tBm (lane 5); DM (lane 6).

a mutant $\mathrm{tBmR}$ (Table 2), which restored dodecamerization (Figure 3) and DNA binding ability (Supporting Information Figure S5B). A mutant, with replacement of Phe (F) to Tyr (Y), showed similar characteristics as that of wild type.

A

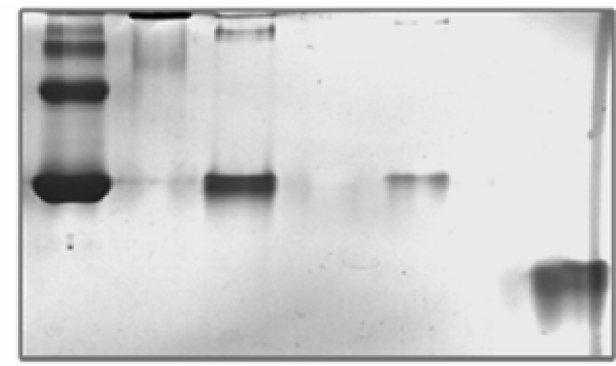

a b c d
Double Amino Acid Mutant at the Trimeric Interface, DM, Resulted in a Native Dps Monomer. Interestingly, the purified double mutant at trimeric interface (DM) generated a heterogeneous protein profile in native PAGE electrophoresis (Figure 4, lane 6). E146A mutation partially disrupted the salt bridge cluster, as discussed before. However, from the results obtained with $\mathrm{tBm}$ mutant, $\mathrm{F}$ to $\mathrm{E}$ mutation at 47 th position completely destabilizes the $\mathrm{tB}$ interface (Figure 1d) formation. Therefore, when a double mutant was generated, only one trimeric form having a weak salt bridge interaction (tA form) is expected to exist in solution. As it is known that increase of ionic strength in solution could preferably disrupt side chain interaction, ionic or electrostatic in nature (21), we further tried to purify this DM mutant under high $\mathrm{NaCl}$ concentration (1 M instead of $500 \mathrm{mM}$ ) in the buffers. As is evident from Figure 5A, lane "e", the purification of DM under high salt concentration resulted in a clean monomer band of the protein in the absence of any denaturant like SDS. Hence, DM is a Dps monomer in its native form. Characterization of this monomer resulted in a functionally inactive Dps, incapable of doing ferroxidation, dodecamerization or DNA binding (Supporting Information Table 2). The purity and homogeneity of all the other single mutants as well as the native protein under $1 \mathrm{M} \mathrm{NaCl}$ purification conditions were also checked. As shown in Figure 5B (lanes $2-8$ ), none of them showed any altered oligomerization profile either. The molecular weights of all the mutants were confirmed through gel filtration chromatography (Figure 6; also see Supporting Information Table 1A).

We also explored the possible existence of other interfaces in $\mathrm{tA}$ or $\mathrm{tB}$ trimers. However, further studies revealed that no other interfaces are present in trimers which may help in dodecamerization.

\section{DISCUSSION}

The major aim of the present work is to explore the molecular mechanism guiding the in vitro oligomerization of Dps protein from M. smegmatis (Ms-Dps) as a function of amino acid mutations at the interfaces inside the macromolecule. We have earlier reported the significance of the temperature induced dodecamerization of the protein, which is directly associated with its bimodal functionality toward DNA protection and ferroxidation (12). This is a unique characteristic of Ms-Dps.

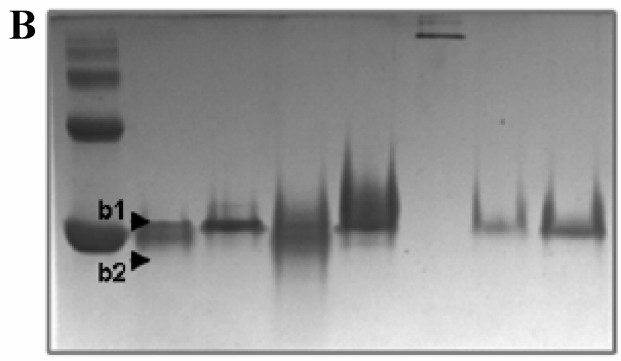

$\begin{array}{lllllll}\mathrm{NaCl}(\mathrm{M}) & 0.5 & 1 & 0.5 & 1 & 0.5 & 1\end{array}$

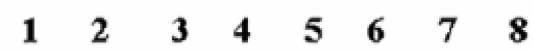

FIGURE 5: Purification profile (10\% native-PAGE) of native Dps and the trimeric interface mutants tAm, tBm and DM under 0.5 and $1 \mathrm{M}$ $\mathrm{NaCl}$ salt conditions at $4{ }^{\circ} \mathrm{C}$ (A) BSA (lane a); horse spleen ferritin (lane b); native Dps purified at $0.5 \mathrm{M} \mathrm{NaCl}$ condition (lane c); native Dps purified at $1 \mathrm{M} \mathrm{NaCl}$ condition (lane d); DM purified at $1 \mathrm{M} \mathrm{NaCl}$ condition (lane e). (B) BSA (lane 1); native Dps (lanes 2, 3); tAm (lane 4, 5); horse spleen ferritin (lane 6); tBm (lanes 7, 8). 

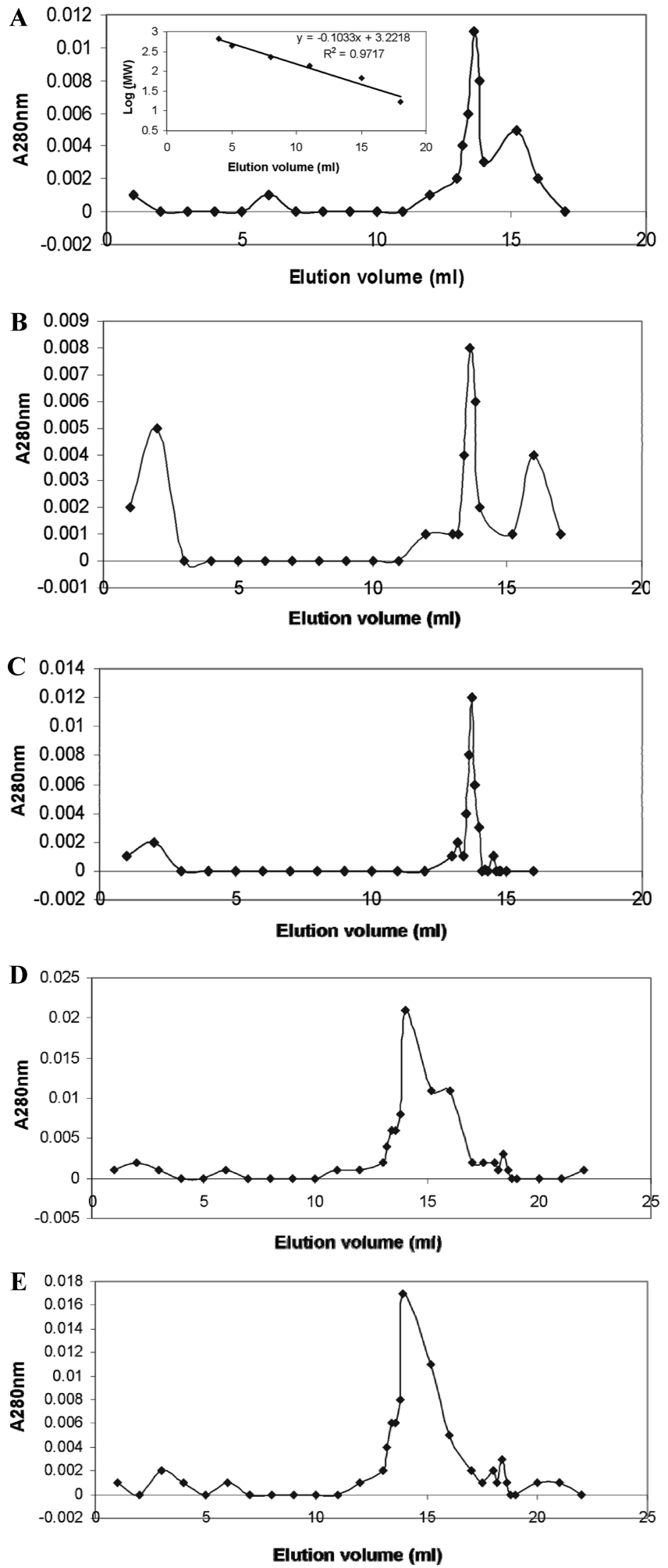

FIGURE 6: Gel filtration profile of native Dps and its single and double amino acid mutants. (A) native Dps (trimer); (B) tAm (trimer); (C) tBm (trimer); (D) tBmR (trimer); (E) DM (monomer). The dodecamers of native, tAm and $\mathrm{tBmR}$ mutants were also checked similarly using the same gel filtration column. $X$ axes represent the elution volume in $\mathrm{mL}$ and $Y$ axes represent the UV absorbance at $280 \mathrm{~nm}$ in each case. The molecular weight calibration curve is shown in $\mathrm{A}$ as an inset, where the $X$ axis represents the elution volume in $\mathrm{mL}$ and the $Y$ axis represents the $\log$ MW.

The trimeric interfaces $\mathrm{tA}$ and $\mathrm{tB}$ obtained from the crystal structure of MsDps are also present in the Dps from Listeria innocua (4). In this work they have generated site directed amino acid mutants at a salt bridge interface to obtain the natural mutant from Listeria monocytogenes. Since the oligomers can be stabilized by a variety of ways, such as the formation of salt bridges, hydrophobic interactions, we have adopted a method of identifying the interface residues from the protein structure networks, which can capture the local details of interactions in the global structural context. We have also identified clusters of amino acids at different interfaces of MS-Dps and have predicted key residues for mutation(s) that can probably disrupt the interfaces. Based on these predictions, single and double mutants are constructed through site directed mutagenesis at both $\mathrm{tA}$ and $\mathrm{tB}$ interfaces and named as tAm (E146A) and tBm (F47E) respectively. Both the mutants are characterized with respect to in vitro dodecamerization and bimodal activity. Though it is expected that disruption of one of the clusters in tA interface (Figure 1a, in "green" and "blue") by mutating the buried glutamate residue (E) at 146th position will impair the trimer formation, to our surprise, we obtain a trimer in this case (Figure 2, lane 3). Furthermore, it is also capable of forming a dodecamer (Figure 2, lane 7), as well as the bimodal activity is also unaltered (see Supporting Information Figures S3-S5). Hence, we propose that tAm is actually a prototype of the native Dps itself. Similarly, tBm was also purified as a trimer (Figure 2, lane 4). However, interestingly, this mutant was found to be incapable of forming a dodecamer under optimum conditions (Figure 2, lane 8).

A $10 \%$ native PAGE analysis of Dps shows two trimeric bands with differential mobility in the gel, as depicted in Figure 5B, lane 2 as "b1" and "b2", at a lower protein concentration (at a higher concentration of the protein load, both the bands appear to merge together). From the experimental results obtained in the present study we would like to propose that these two bands are due to two trimeric interfaces, $t A$ and $t B$ Dps proteins and based on this we have proposed a model depicting the trimer to dodecamer conversion in Ms-Dps as shown in Figure 7. Perhaps we can argue that the E146A mutation did not completely disrupt the tA interface, but it was partially weakened, as the salt bridge cluster (shown in "green" in Figure 1a) is disrupted. However the other independent cluster (shown in "blue" in Figure 1a) remained unperturbed. Thus, it is likely that tAm exhibits similar property as that of the native protein, where both the interfaces are still present (Figure 7).

We have shown with gel filtration analysis that these lower forms match with dimeric Dps molecules, and their absence in tAm may be the effect of disruption of a dimeric interface between two monomers due to the conversion of $\mathrm{E}$ to A(Figure 1a) instead of a true trimeric interface. It appears that this mutant directly converts itself into a more stable dodecamer without any intermediate dimer formation, which is one of the probable mechanisms for native Dps to dodecamerize (Figure 7, compare native Dps and tAm).

On the other hand, the tBm mutant becomes more significant as it cannot convert itself into a dodecamer like the native or the tAm Dps. This can be rationalized by assuming that the mutation from Phe47 (F) to Glu47 (E) in the unique hydrophobic cluster in $\mathrm{tB}$ interface (Figure 1c, shown in "blue") is deleterious to the formation of the Dpslike trimer interface, resulting in the presence of only one trimer interface $(\mathrm{tA})$ in solution. This is a very stable trimer 

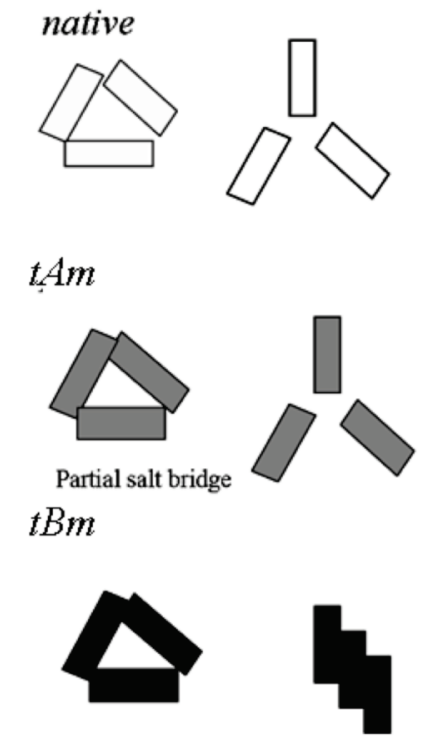

$D M$
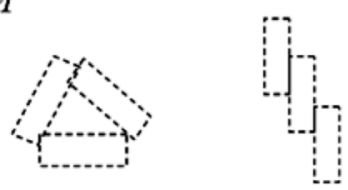
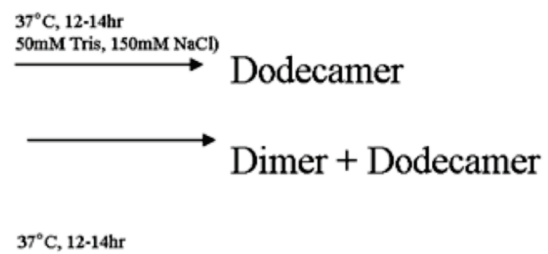

$50 \mathrm{mM}$ Tris, $150 \mathrm{mM}$ NaCl)
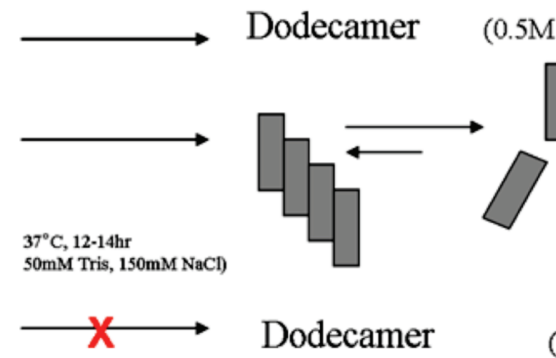

Dodecamer

$(0.5 \mathrm{M}$ and $1 \mathrm{M} \mathrm{NaCl})$

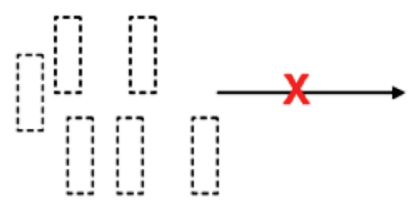

\section{Dodecamer}

$(1 \mathrm{M} \mathrm{NaCl})$

FIGURE 7: Probable model depicting the in vitro oligomerization of Dps protein in solution. Each bar represents monomer chains of the protein: native Dps (white); tAm (gray); tBm (black); DM (dashed).

with strong salt bridge clusters. It cannot fold into a dodecamer further, either due to its high thermodynamic stability or due to a high kinetic energy barrier to go to a dodecamer in the absence of the tB form, which perhaps is the rate limiting step for this in vitro protein-protein assembly. F47 residue is primarily important for building up the tB interface, and this in turn is important to fold into the dodecamer in the presence of the other form. This is further supported by constructing revert mutant $\mathrm{tBmR}$, by replacing " $\mathrm{E}$ " residue in $\mathrm{tBm}$ by another hydrophobic amino acid with aromatic ring, e.g. "Y" at the 47 th position. As expected, restoration of the hydrophobic core in the tB cluster in $\mathrm{tBmR}$ concomitantly restores the dodecamerization ability of tBm mutant (Figure 3, lane 5), similar to that of the native Dps (compare lane 6 in Figure 2). It provides a new insight into the significance of a hydrophobic interaction among the amino acid side chains (as in tB) vis-à-vis salt bridge interaction (as in tA), in stabilizing different oligomeric interfaces in a multimeric protein. Sequence alignment has shown that the F47 residue is highly conserved in all sequences of the Dps family of proteins in both Gram negative and Gram positive bacteria (9). Hence our results support the significance of this conserved residue in MsDps toward the formation of the $\mathrm{tB}$ interface and its importance to form the dodecamer with the tA counterpart.

Double mutant $(\mathrm{DM})$, generated at the trimeric interface clusters, was a monomer incapable of oligomerization and inactive with respect to both ferroxidation and DNA binding activity. We can achieve a clean monomer only at high salt purification condition. This may be due to the complete disruption of a weaker tA interface and the absence of the $\mathrm{tB}$ interface due to $\mathrm{F}$ mutation, leaving only the monomers in solution (Figure 7). We have also shown that purification of native, tAm as well as tBm under very high salt condition (up to $1 \mathrm{M} \mathrm{NaCl}$ ) does not alter the purification profile (Figure 5B).

To summarize, both trimers probably exist in solution. Both of them are essential for dodecamer formation. However the tB interface is the rate determining step of oligomerization. Importantly, hydrophobicity and aromatic stacking play an important role in stabilizing the interface cluster in Dps. We believe that the present study will provide some newer insights on the stabilizing interactions in symmetric multimeric proteins at the molecular level.

\section{SUPPORTING INFORMATION AVAILABLE}

Figures S1-S5 and tables of data on gel filtration chromatography, dynamic light scattering, and functional characterization of mutants. This material is available free of charge via the Internet at http://pubs.acs.org.

\section{REFERENCES}

1. Grueninger, D., Treiber, N., Zieglar, M. O. P., Koetter, J. W. A., Schulze, M. S., and Schulz, G. E. (2008) Designed Protein-Protein Association. Science 319, 206-209.

2. Frederick, K. K., Marlow, M. S., Valentine, K. G., and Wand, J. (2007) Conformational entropy in molecular recognition by proteins. Nature 448, 325-330.

3. Cole, C., and Warwicker, J. (2002) Side-chain conformational entropy at protein-protein interfaces. Protein Sci. 11, 2860-2870.

4. Bellapadrona, G., Chiaraluce, R., Consalvi, V., Ilari, A., Stefanini, S., and Chiancone, E. (2007) The Mutations Lys $114 \rightarrow$ Gln and Asp $126 \rightarrow$ Asn Disrupt an Intersubunit Salt Bridge and Convert Listeria innocua Dps Into its Natural Mutant Listeria monocytogenes Dps. Effects on Protein Stability and Low pH. Proteins 66, 975-983.

5. Bajaj, K., Chakrabarti, P., and Varadarajan, R. (2005) Mutagenesisbased definitions and probes of residue burial in proteins. Proc. Natl. Acad. Sci. U.S.A. 102, 16221-16226.

6. Bajaj, K., Madhusudan, M. S., Adkar, B. V., Chakrabarti, P., Ramakrishnan, C., Sali, A., and Varadarajan, R. (2007) Stere- 
ochemical Criteria for Prediction of the Effects of Proline Mutations on Protein Stability. PLoS Comput. Biol 3 (12), e241.

7. Baskakov, I. V., Aagaard, C., Mehlhorn, I., Wille, H., Groth, D., Baldwin, M. A., Prusiner, S. B., and Cohen, F. E. (2000) SelfAssembly of Recombinant Prion Protein of 106 Residues. Biochemistry 39, 2792-2804.

8. Ortells, M. O., and Barrantes, G. E. (2008) A model for the assembly of nicotinic receptors based on subunit-subunit interactions. Proteins 70, 473-488.

9. Gupta, S., Pandit, S. B., Srinivasan, N., and Chatterji, D. (2002) Proteomics analysis of carbon-starved Mycobacterium smegmatis: induction of Dps-like protein. Protein Eng. 15, 503-512.

10. Roy, S., Gupta, S., Das, S., Sekar, K., Chatterji, D., and Vijayan, M. (2003) Crystallization and preliminary X-ray diffraction analysis of Mycobacterium smegmatis Dps. Acta Crystallogr., Sect. D: Biol. Crystallogr. 59, 2254-2256.

11. Roy, S., Gupta, S., Das, S., Sekar, K., Chatterji, D., and Vijayan, M. (2004) X-ray analysis of Mycobacterium smegmatis Dps and a comparative study involving other Dps and Dps-like molecules. J. Mol. Biol. 339, 1103-1113.

12. Gupta, S., and Chatterji, D. (2003) Bimodal protection by Mycobacterium smegmatis DNA-binding protein from stationary phase cells. J. Biol. Chem. 278, 5235-5241.

13. Kannan, N., and Vishveshwara, S. (1999) Identification of Sidechain Clusters in Protein Structures by a Graph Spectral Method. J. Mol. Biol. 292, 441-464.

14. Kannan, N., Chander, P., Ghosh, P., Vishveshwara, S., and Chatterji, D. (2001) Stabilizing interactions in the dimer interface of $\alpha$-subunit in Escherichia coli RNA polymerase: A graph spectral and point mutation study. Protein Sci. 10, 46-54.

15. Brinda, K. V., and Vishveshwara, S. (2005) A Network Representation of Protein Structures: Implications for Protein Stability. Biophys. J. 89, 4159-4170.

16. Bogan, A. A., and Thorn, K. S. (1998) Anatomy of Hot Spots in Protein Interfaces. J. Mol. Biol. 280, 1-9.

17. Chothia, C., and Janin, J. (1975) Principles of protein-protein recognition. Nature 256, 705-708.
18. Jain, V., Saleem-Batcha, R., China, A., and Chatterji, D. (2006) Molecular disection of the mycobacterial stringent response protein Rel. Protein Sci. 15, 1449-1464.

19. Brinda, K. V., and Vishveshwara, S. (2005) Oligomeric protein structure networks: insights into protein-protein interactions. $B M C$ Bioinf. 6, 296.

20. Gage, M. J., and Robinson, A. S. (2003) C-terminal hydrophobic interactions play a critical role in oligomeric assembly of the P22 tailspike trimer. Protein Sci. 12, 2732-2747.

21. Peyser, Y. M., Ajtai, K., Burghardt, T. P., and Muhlrad, A. (2001) Effect of Ionic Strength on the Conformation of Myosin Subfragment 1-Nucleotide Complexes. Biophys. J. 81, 1101-1114.

22. Chelli, R., Gervasio, F. L., Procacci, P., and Schettino, V. (2002) Stacking and T-shape Competition in Aromatic-Aromatic Amino Acid Interactions. J. Am. Chem. Soc. 124, 6133-6143.

23. Shenoy, A. R., and Visweswariah, S. (2003) Site-directed mutagenesis using a single mutagenic oligonucleotide and DpnI digestion of template DNA. Anal. Biochem. 319, 335-336.

24. Dezs"o, Z., and Barabási, A. L. (2002) Halting viruses in scalefree networks. Phys. Rev. E 65, 055103(R).

25. Barabási, A. L., and Oltvai, Z. N. (2004) Network Biology: Understanding The Cell's Functional Organization. Nat. Rev. Genet. $5,101-113$

26. Albert, R. (2005) Scale-free networks in cell biology. J. Cell Sci. $118,4947-4957$

27. Candia, J. (2007) Irreversible opinion spreading on scale-free networks. Phys. Rev. E 75, 026110.

28. Vermeirssen, V., Barrasa, M. I., Hidalgo, C. A., Babon, J. A., Sequerra, R., Doucette-Stamm, L., Barabási, A. L., and Walhout, A. J. M. (2008) Genome Res. 17, 1061-1071.

29. Cormen, T. H., Stein, C., Rivest, R. L., and Leiserson, C. E. (2001) Introduction to Algorithms, McGraw-Hill Higher Education, New York.

30. Chowdhury, R. P., and Chatterji, D. (2007) Estimation of Förster's distance between two ends of Dps protein from mycobacteria: Distance heterogeneity as a function of oligomerization and DNA binding. Biophys. Chem. 128, 19-29.

BI801158E 